\title{
Asociación de mortalidad, deformación longitudinal global y circunferencial del ventrículo izquierdo en pacientes con sepsis en terapia intensivall
}

\author{
Ahtziri Yunuén Rodríguez Santos, ${ }^{*}$ Andrea Rugenio Cabrera, ${ }^{*}$ Julio Sauza Sosa, ${ }^{*}$ \\ Juvenal Franco Granillo, ${ }^{*}$ Janet Aguirre Sánchez, ${ }^{\star}$ Gilberto Camarena Alejo*
}

\section{RESUMEN}

Introducción: La sepsis es una de las principales causas de morbimortalidad en la unidad de terapia intensiva (UTI), y al vincularse con miocardiopatía asociada a sepsis (SCM), empeora el pronóstico; se puede presentar hasta en $68 \%$ de los pacientes. Se han propuesto parámetros por ecocardiografía (ECO) útiles para la valoración de la función sistólica del ventrículo izquierdo (VI), como la deformación global (STRAIN-GL) y deformación circunferencial (STRAIN-C) del ventrículo izquierdo. En este estudio se valoró la asociación de los mismos con la mortalidad en los pacientes con sepsis en UTI.

Material y métodos: Se realizó un estudio transversal, prospectivo, de cohorte en pacientes con sepsis ingresados en la UTI de un centro hospitalario de la Ciudad de México, evaluados del primero de enero al 31 de julio 2018; se tomó ECO transtorácico dentro de las primeras 24 horas del diagnóstico de sepsis.

Resultados: Se incluyeron 30 pacientes, 17 masculinos $(56.7 \%)$, con una mediana de edad de 68.5 años (RIQ 56-84) y una mediana de estancia intrahospitalaria de 12 días (RIQ 7-17). La tasa de defunción fue de $23.3 \%$. La duración media de estancia en la UCI fue de 19.1 días (3-185 días). Valorado de manera independiente por CS, se encontró que $36 \%$ de la población presentó disfunción sistólica del VI, mientras que valorado por GLS, se obtuvo $40 \%$ de la población. Al comparar los no sobrevivientes y los sobrevivientes, no hubo diferencias demográficas significativas; se observó una diferencia de proporciones en el número de pacientes con diabetes. Se registraron diferencias en las medianas de PSAP (presión sistólica de la arteria pulmonar), VAI (volumen aurícula izquierda), CS y GLS. Al probar la capacidad para discriminar los sobrevivientes contra los no sobrevivientes, se observó que el CS fue marginalmente superior que el GLS, APACHE (Acute Physiology, Age and Chronic Health Evaluation) II, SAPS (Simplified Acute Physiology Score) II y SOFA (evaluación del fallo orgánico secuencial). Al probar la capacidad de CS y GLS para discriminar la estancia hospitalaria mayor a siete días, se pudo ver que las medidas ecocardiográficas fueron superiores. Se seleccionaron puntos de corte para la discriminación de sobrevivientes contra no sobrevivientes mediante el análisis de sensibilidad y especificidad para las siguientes medidas ecocardiográficas: CS $\geq-15.1$ (S: $71.43 \%$, E: 83.33\%, LR(+) 4.3, LR(-) 0.34) y GLS $\geq-15.4$ (S: $85.71 \%, E: 73.91 \%$, LR(+) 3.3, LR(-) 0.19). En el análisis multivariado se encontró que un $\mathrm{CS} \geq-15.1$ fue predictivo de mortalidad durante la hospitalización en el periodo y la población de estudio, ajustado para otras variables ecocardiográficas como $\mathrm{FEVI}<55 \%$, GLS $\geq-15.4$ y confusores como sexo [RM = 10.23 (IC 95\%, 1.01-103.2), p < 0.049]. En modelos de regresión lineal no se encontraron variables ecocardiográficas predictoras de los días de estancia hospitalaria en el periodo y la población de estudio.

Conclusiones: El desarrollo de nuevas técnicas de ECO, como el speckle tracking echocardiography (STE), ha facilitado la capacidad de evaluar la función del VI mediante la evaluación cuantitativa de la deformación miocárdica; aunque son técnicas complejas, si se realizan e interpretan de forma apropiada, son de gran utilidad en diferentes condiciones clínicas. La disfunción miocárdica (MD) puede ser un marcador temprano de mortalidad en pacientes con sepsis; obtenida mediante la medición de GLS y CS, podría ser un predictor pronóstico confiable de los resultados de los pacientes en la UTI, pudiendo también potencializar escalas como el APACHE II, SOFA y SAPS II para permitir la identificación temprana de pacientes sépticos de alto riesgo. Aún existen ciertos obstáculos para la aplicación clínica regular de GLS y CS en pacientes sépticos en la UTI. El límite de GLS óptimo para la predicción de la mortalidad en estos pacientes sigue siendo incierto, y las diferencias intrínsecas entre las poblaciones podrían contribuir a las diferencias observadas; sin embargo, se sabe que es un parámetro eficaz para la cuantificación de la función ventricular izquierda, más sensible incluso que la FEVI por ECO bidimensional, y depende

\footnotetext{
" Ganador del Premio Académico «Mario Shapiro». Primer lugar.

* Centro Médico ABC.
}

Recepción: 27/08/2018. Aceptación: 22/11/2018.

Este artículo puede ser consultado en versión completa en http://www.medigraphic.com/medicinacritica relativamente menos del operador y las condiciones de carga. Los resultados actuales deben confirmarse en estudios adicionales a gran escala y multicéntricos. Por ello, aún permanece en el campo de la investigación clínica para los pacientes en estado crítico.

Palabras clave: Miocardiopatía, deformación global longitudinal, deformación circunferencial.

\section{SUMMARY}

Introduction: Sepsis is one of the main causes of morbidity and mortality in the ICU, and when associated with SCM, the prognosis worsens; it can occur in up to $68 \%$ of patients. ECHO parameters have been proposed useful for the assessment of the LV systolic function, such as STRAIN-GLS and STRAIN-CS. In this study, the association of these with mortality in patients with sepsis in the ICU was assessed.

Material and methods: A prospective, cross-sectional, cohort study was conducted in patients with sepsis admitted to the ICU of a hospital center in Mexico City from January $1^{\text {st }}$ to July $31^{\text {st }}$, 2018. Transthoracic ECHO was taken within the first 24 hours of the diagnosis of sepsis.

Results: Thirty patients were included, 17 were male (56.7\%); the median age was 68.5 years (RIQ 56-84); the median hospital stay was 12 days (RIQ 7-17). The death rate was of $23.3 \%$. The average length of stay in the ICU was 19.1 days (3-185 days). Independently assessed by CS, it was found that $36 \%$ of the population presented LV systolic dysfunction, while assessed by GLS, it was $40 \%$. When comparing non-survivors and survivors, there were no significant demographic differences; a difference of proportions was observed in the number of patients with diabetes. Differences in medians of systolic pulmonary artery pressure (SPAP), VAI, CS and GLS were observed. When testing the ability to discriminate survivors against non-survivors, it was found that CS was marginally superior to GLS, APACHE (Acute Physiology Age and Chronic Health Evaluation) II, SAPS (Simplified Acute Physiology Score) II, and SOFA (Sequential Organ Failure Assessment). When testing the ability of CS and GLS to discriminate hospital stay greater than seven days, it was observed that the echocardiographic measures were superior. Cut-off points were selected for discrimination of survivors against non-survivors by analyzing sensitivity and specificity for the following echocardiographic measurements: $C S \geq-15.1$ (S: $71.43 \%, E: 83.33 \%, L R(+) 4.3, L R(-) 0.34$ ) and $G L S \geq-15.4$ (S: $85.71 \%$, E: $73.91 \%, L R(+) 3.3, L R(-) 0.19)$. In the multivariate analysis, it was found that a $C S \geq-15.1$ was predictive of mortality during hospitalization in the study period and population, adjusted for other echocardiographic variables such as $L V E F<$ $55 \%, G L S \geq-15.4$ and confounders such as sex [RM $=10.23$ (95\% Cl, 1.01103.2), $p<0.049$ ]. In linear regression models, no predictive echocardiographic variables were found for days of hospital stay in the study period and population. Conclusions: The development of new ECHO techniques such as speckle tracking echocardiography (STE) has facilitated the ability to evaluate LV function through the quantitative evaluation of myocardial deformation; although they are complex techniques, if performed and interpreted appropriately, they are very useful in different clinical conditions. MD can be an early marker of mortality in patients with sepsis; obtained by measuring the GLS and CS, it could be a reliable predictor of the outcomes of patients in the ICU, and it can also potentiate scales such as APACHE II, SOFA and SAPS II to allow early identification of septic patients at high risk. There are still some obstacles to the regular clinical application of GLS and CS in septic patients in the ICU. The optimal GLS limit for the prediction of mortality in these patients remains uncertain, and the intrinsic differences between the populations could contribute to the observed differences. However, it is known to be an effective parameter for the quantification of left ventricular function, even more sensitive than the LVEF by bidimensional ECHO, depending relatively less on the operator and loading conditions. Current results should be confirmed in additional large-scale and multi-center studies. Therefore, it still remains in the field of clinical research for patients in critical condition.

Key words: Cardiomyopathy, longitudinal global deformation, circumferential deformation.

RESUMO

Introdução: A sepse é uma das principais causas de morbidade e mortalidade na UTI e quando associada à SCM piora o prognóstico, pode ocorrer em até 
$68 \%$ dos pacientes. Os parâmetros do ECO têm sido propostos como úteis para a avaliação da função sistólica do VE, como STRAIN-GLS e STRAIN-CS, neste estudo foi avaliada a associação destes com a mortalidade em pacientes com sepse na UTI.

Material e métodos: Realizou-se um estudo prospectivo, transversal, de coorte de um centro hospitalar em pacientes com sepse internados na UTI na Cidade do México, avaliados de 1 de janeiro a 31 de julho de 2018. Foi realizado ECO transtorácico nas primeiras 24 horas do diagnóstico de sepse.

Resultados: Foram incluídos 30 pacientes, sendo 17 do sexo masculino (56.7\%), idade média 68.5 anos (RiQ 56 - 84), internação média de 12 dias (RIQ 7-17). Com uma taxa de mortalidade de $23.3 \%$. O tempo médio de permanência na UTI foi de 19.1 dias (3-185 dias). Avaliada independentemente pelo CS, constatou-se que $36 \%$ da população apresentava disfunção sistólica do VE, enquanto avaliada por GLS obtiveram $40 \%$ da população. Quando comparados não-sobreviventes e sobreviventes não houve diferenças demográficas significativas, observou-se uma diferença de proporções no número de pacientes com diabetes. Foram observadas diferenças nas medianas de PSAP, VAI, CS e GLS. Ao testar a capacidade de discriminar sobreviventes versus não sobreviventes, verificou-se que o CS foi marginalmente superio ao GLS APACHE II, SAPS I/ e SOFA. Ao testar a capacidade de CS e GLS em discriminar a permanência hospitalar superior a 7 dias, observou-se que as medidas ecocardiográficas foram superiores. Foram selecionados pontos de corte para a discriminação de sobreviventes versus não sobreviventes analisando a sensibilidade e especificidade para as seguintes medidas ecocardiográficas: $E C \geq-15.1$ (S: $71.43 \%, E: 83.33 \%, L R(+)$ 4.3, LR (-) 0.34) e GLS $\geq-15.4$ (S: $85.71 \%, E: 73.91 \%, L R(+)$ 3.3, LR (-) 0.19). Na análise multivariada, verificou-se que um $C S \geq-15.1$ foi preditivo de mortalidade durante a internação no período e população do estudo, ajustado para outras variáveis ecocardiográficas como FEVE $<55 \%$, GLS $\geq-15.4$ e confundidores como sexo. [RM = 10.23 (IC 95\%, 1.01-103.2), $p<0.049]$. Nos modelos de regressão linear, não foram encontradas variáveis ecocardiográficas preditivas para os dias de internação hospitalar no período e população do estudo.

Conclusões: $O$ desenvolvimento de novas técnicas de ECO, como STE facilitou a capacidade de avaliar a função do VE através da avaliação quantitativa da deformação miocárdica, embora sejam técnicas complexas, $s$ se realizam e interpretam de forma adequada, são muito úteis em diferentes condições clínicas. A MD pode ser um marcador precoce de mortalidade em pacientes com sepse, obtida pela mensuração da GLS e CS poderia ser um preditor prognóstico confiável dos resultados de pacientes na UTI, e também pode potencializar escalas como APACHE II, SOFA e SAPS II para permitir a identificação precoce de pacientes sépticos de alto risco. Existem ainda alguns obstáculos para a aplicação clínica regular de GLS e CS em pacientes sépticos na UTI, apesar de ser um estudo à beira do leito, é necessário levar em conta a gravidade da doença e comorbidades prévias. O limite ideal de GLS para a predição de mortalidade nesses pacientes permanece incerto $e$ as diferenças intrínsecas entre as populações poderiam contribuir para as diferenças observadas, porém é sabido que é um parâmetro efetivo para a quantificação da função ventricular esquerda, mais sensível que a FEVE por ECO bidimensional, dependem relativamente menos do operador e das condições de carga. Os resultados atuais devem ser confirmados em estudos adicionais em larga escala e multicêntricos. Por isso, ainda permanece no campo da pesquisa clínica para pacientes em estado crítico.

Palavras-chave: Cardiomiopatia, deformação global longitudinal, deformação circunferencial.

\section{INTRODUCCION}

La disfunción miocárdica (MD), al encontrarse asociada con sepsis, empeora el pronóstico de los pacientes en terapia intensiva,${ }^{1-3}$ ocasionado por la liberación de mediadores proinflamatorios y moléculas de señalización, con la liberación concomitante de mediadores antiinflamatorios (MAl), ${ }^{4}$ lo que conduce a falla multiorgánica (FMO) y muerte en algunos pacientes. ${ }^{5}$ En la actualidad, se sabe que los trastornos en la fisiología de los cardiomiocitos desempeñan un papel importante a nivel de la microcirculación, ocasionado por moléculas inflamatorias mediante efectos pleiotrópicos. ${ }^{5-8}$ Existe disfunción del miocardio a nivel mitocondrial en pacientes sépticos causada por alteraciones del calcio secundarias al incremento del estrés oxidativo; al tener altas demandas de energía del tejido cardiaco, no son capaces de compensar. De manera simultánea, hay daño provocado por cambios en la permeabilidad endotelial, con subsecuente edema, paso de neutrófilos al intersticio, formación de depósitos de fibrina y activación de la cascada de coagulación, lo que conduce al mal funcionamiento del aparato contráctil, alteraciones en la regulación autónoma, disminución de la expresión de los receptores adrenérgicos y resistencia a las catecolaminas endógenas. ${ }^{9,10}$

Los dos objetivos principales en el tratamiento de la sepsis son el manejo de la infección y la optimización de la función hemodinámica (mediante la administración intravenosa de soluciones cristaloides y vasopresores). El aumento de la precarga (con el objetivo de elevar el CO a través del mecanismo de Frank-Starling) es uno de los métodos más utilizados para la reanimación en sepsis, pero depende del estado funcional del corazón; es decir, un corazón con una contractilidad intrínseca deficiente puede aumentar el CO si la RVS (resistencia vascular sistémica) es baja, aparentando una función normal (aunque el rendimiento sistólico se encuentre afectado); por lo tanto, esta disfunción se manifiesta cuando la RVS vuelve a la normalidad, ya sea por recuperación de la sepsis o uso de vasopresores. ${ }^{11-15}$ Además de las alteraciones en la precarga, en los pacientes sépticos también existen cambios en la postcarga que afectan la capacidad del corazón para transportar sangre a los tejidos periféricos. A pesar de las múltiples propuestas y publicaciones en los últimos años, el adecuado monitoreo hemodinámico aún continúa siendo un tema de discusión relevante para facilitar la reanimación en etapas tempranas.

La miocardiopatía asociada a sepsis (SCM) se puede presentar en 20 a $68 \%$ de los pacientes con sepsis y aumenta la mortalidad en más de dos veces en comparación con quienes tuvieron insuficiencia orgánica, pero sin aparente afección cardiaca. ${ }^{16,17}$ La definición original fue descrita por Parker y sus colaboradores tres años más tarde, al utilizar catéteres de arteria pulmonar e imágenes de radionúclidos. Definieron la SCM con CO alto, baja RVS y adecuado mantenimiento del volumen sistólico (VS), siempre y cuando el paciente hubiera recuperado volumen adecuadamente. Reportaron que los sobrevivientes del choque séptico aumentaron los volúmenes del ventrículo izquierdo $(\mathrm{VI})$ al final de la diástole y fracción de eyección del ventrículo izquierdo (FEVI) reducida $<40 \%$, mientras que los no supervivientes tenían mayor FEVI y volúmenes ventriculares normales; demostraron la reversibilidad en alrededor de siete a 10 días. ${ }^{18}$ En la actualidad, la SCM está definida como la disfunción sistólica o diastólica del VI o del ventrículo derecho (VD) de nuevo inicio, evidenciada por ecocardiografía (ECO) en pacientes con diagnóstico de sepsis, de etiología multifactorial, ${ }^{19}$ 
con la capacidad de reversibilidad (comportándose como miocardio en «hibernación»). 8,9,20 Es impulsada por una cascada de procesos celulares y moleculares, por lo que a nivel histológico, típicamente se observa edema intersticial significativo con grandes cantidades de glóbulos blancos (neutrófilos y macrófagos) en el mismo, sin evidencia de destrucción de las miofibrillas. La etiología más estudiada de la SCM se divide en tres rubros: por inestabilidad hemodinámica, cambios en el flujo sanguíneo y toxicidad directa del miocardio, ya comentada. La SCM se produce con frecuencia en fases iniciales de la sepsis y puede afectar ambos ventrículos, manifestándose como un desequilibrio entre el suministro de oxígeno $\left(\mathrm{DO}_{2}\right)$ y el consumo de oxígeno $\left(\mathrm{VO}_{2}\right)$ y causando, en ocasiones, saturación de oxígeno venosa central baja $\left(\mathrm{ScvO}_{2}\right)$ e incremento en el lactato. Al guiar la reanimación de los pacientes sépticos mediante estos parámetros (lactato y $\mathrm{ScvO}_{2}$ ), se requiere la presencia de un catéter venoso central (CVC) - con riesgo de complicaciones-, por lo que se ha cuestionado su utilidad de manera aislada para la reanimación, teniéndose en cuenta nuevas opciones como la ECO, que no es invasiva, puede ser a la cabecera del paciente y, además de predecir respuesta a volumen, ayuda a valorar la función sistólica y diastólica. ${ }^{21}$

En la actualidad existen múltiples técnicas no invasivas de imagen para la evaluación de la función sistólica del VI, como la resonancia magnética cardiaca (RMC), la tomografía computarizada multicorte cardiaca (TACmc), la ventriculografía isotópica (Vist) y la ECO, entre otras. La mayoría de los métodos convencionales presentan baja sensibilidad para la valoración exhaustiva de la contractilidad miocárdica, ya que no detectan de manera precoz pequeños cambios de contractilidad o alteraciones, lo que representa múltiples limitaciones para utilizarlos en pacientes hospitalizados en las áreas críticas. ${ }^{22}$

En las últimas décadas, el uso de la ECO ha presentado gran auge en las áreas de cuidados críticos, ya que es una medición no invasiva y rápida que se puede realizar a la cabecera del paciente; sin embargo, gracias a los parámetros tradicionales como la FEVI ([FEVI $=(($ LVEDV-LVESV)/LVEDV) $X 100]$ (tomada como valor anormal $<52 \%$ en hombres y $<54 \%$ en mujeres), que es una medida imperfecta - ya que depende tanto de la contractilidad como de las condiciones de carga del $\mathrm{VI}-$, ha demostrado poca aplicabilidad y valor pronóstico en pacientes con SCM, ${ }^{23}$ por lo que su interpretación se debe mantener con reservas. En 2014 se realizó un metaanálisis que incluyó a 585 estudios; encontraron que la FEVI disminuida tenía una sensibilidad de $52 \%$ (IC 95\% 29-73\%) y una especificidad de $63 \%$ (IC 95\% $53-71 \%$ ) para la mortalidad; no era predictor de mortalidad sensible o específico. ${ }^{24}$ Aún se siguen realizando estudios que incluyen valoración de la FEVI en pacientes sépticos, como el de Boissier y su grupo, que demostraron una relación inversa entre FEVI y RVS en pacientes sépticos. ${ }^{25}$

Se han propuesto otros parámetros ecocardiográficos que en ciertos pacientes pueden ser útiles para la valoración de la función sistólica del VI, como la deformación longitudinal del ventrículo izquierdo (STRAINL), deformación global del ventrículo izquierdo (STRAIN-GL), deformación radial (STRAIN-R), deformación circunferencial del ventrículo izquierdo (STRAIN-C) y la torsión (t).

Las fibras miocárdicas (FM) se encuentran distribuidas con diferente orientación en los distintos segmentos ventriculares; tienen propiedades de cambio de tamaño y forma constante. Durante la contracción normal, la FM puede cambiar su tamaño simultáneamente en varias direcciones, creando vectores de deformación de forma tridimensional. ${ }^{26}$

En la ECO se puede decir que existen tres patrones de deformación miocárdica durante la sístole: a) longitudinal (se refiere al acortamiento base-ápex), b) circunferencial (las fibras se acortan en el sentido de la circunferencia de la cavidad) y c) radial (se refiere al engrosamiento del epicardio hacia el endocardio; las fibras tienden a converger hacia un punto en el centro del ventrículo). ${ }^{27}$ Además de la deformación mencionada durante esta fase, el VI presenta rotación y torsión (el ápex gira en una dirección y la base gira en dirección contraria). ${ }^{28,29}$ Durante la diástole, el músculo cardiaco en condiciones normales presenta desplazamientos opuestos a los de la sístole; en esta fase, la fibra retorna a su estado original o basal. Estos desplazamientos y deformaciones de la fibra miocárdica pueden estudiarse mediante diferentes métodos como la RM (estándar de oro) y el speckle tracking echocardiography (STE) para obtener la deformación longitudinal del ventrículo izquierdo (STRAIN-LS), deformación global del ventrículo izquierdo (STRAINGLS), deformación radial (STRAIN-R), deformación circunferencial del ventrículo izquierdo (STRAIN-CS) y la torsión ( $\mathrm{t}$ ). El método STE se ha comparado con los datos que se obtienen mediante RM tanto en segmentos miocárdicos normales como anormales; se ha obtenido muy buena correlación $(r=0.87, p<0.001)$ y se ha validado este método ecocardiográfico. ${ }^{30} \mathrm{Es}$ una técnica relativamente nueva, ya que fue desarrollada en el año 2004. Está basada en imágenes de ECO bidimensional archivadas en formato digital (es llamada también estudio «off line»); ${ }^{31,32}$ es capaz de detectar pequeñas diferencias en la textura similares a «huellas» de cada segmento miocárdico, diferenciando con claridad un segmento de otro; una vez que se detecta la «huella» de un segmento miocárdico, se le puede rastrear (tracking) y medir en múltiples momen- 
tos del ciclo cardiaco, y así, calcular su estiramiento o deformación a partir de un punto cero fijado con anterioridad, generando vectores de movimiento y curvas de deformación. ${ }^{33-35}$ The European Association of Cardiovascular Imaging (EACVI) y American Society of Echocardiography (ASE) estandarizaron las técnicas de análisis de la deformación miocárdica usando imágenes de ECO bidimensional y speckle tracking. ${ }^{36}$ La medición a través de Doppler tisular (IDT) requiere imágenes ecocardiográficas $2 \mathrm{D}$ de excelente calidad, con una visualización óptima del tejido miocárdico y el borde del endocardio, ya que depende del ángulo, tiene una relación señal-ruido relativamente baja y requiere experiencia considerable para la interpretación correcta. Por el contrario, la ECO mediante el método STE no depende del ángulo y tiene menos variación interobservador, ya que utiliza un algoritmo a través de un software, el cual extrae el desplazamiento, la velocidad, el SR y la deformación dentro del segmento miocárdico, definido por medio del seguimiento de «huellas». Este método está limitado por su dependencia de la calidad de las imágenes ecocardiográficas; puede existir movimiento fuera del plano por el seguimiento inadecuado del movimiento 3D del corazón, el sombreado acústico, las reverberaciones, y en comparación con el IDT, utiliza tasas de cuadro más bajas (50 a 90 cuadros/s), lo que disminuye su precisión. A pesar de estas limitaciones, el STE es una técnica más simple, reproducible e independiente del ángulo que sirve como la mejor opción para evaluar la función miocárdica regional en la terapia intensiva. ${ }^{37}$ Las mediciones se deben realizar por separado para calcular cada uno de los tipos de deformación. El strain longitudinal y la torsión se estudian desde proyecciones apicales (cuatro, dos cámaras y eje largo apical), mientras que el strain radial y circunferencial se estudian en eje corto paraesternal izquierdo, en un corte que usualmente se hace a nivel de los músculos papilares. ${ }^{38}$

El strain longitudinal mide la deformación en el sentido base-ápex de la cavidad (acortamiento de la cámara): la longitud final de la cavidad o sistólica es menor que la longitud inicial o diastólica, por lo que el porcentaje de la deformación es negativo. ${ }^{22}$ El porcentaje de cambio de tamaño de la fibra se obtiene mediante la longitud de la fibra durante su máximo estiramiento (L1) - tamaño de la fibra en reposo (Lo) entre tamaño de la fibra en reposo (Lo), ${ }^{39-41}$ dígase:

$$
\text { S = L1-Lo/Lo }
$$

Durante la sístole, el VI también se acorta en dirección circunferencial, por lo que resulta un porcentaje de tensión circunferencial negativa. Por el contrario, el engrosamiento radial hace que la longitud sistólica sea mayor que la longitud inicial o diastólica, por lo que la tensión radial es positiva.

El strain y el SR pueden ser evaluados en cada región del ventrículo estudiado (strain regional), y el promedio de estos valores representa el strain longitudinal global (refleja la función ventricular global), siendo el más estudiado clínicamente. En condiciones normales, el valor de la tensión longitudinal global promedio de 17 segmentos vistos desde proyecciones apicales se expresa en porcentaje de cambio de tamaño; ${ }^{39}$ debe ser más negativo que $-18 \%$. Desde 2009 se sabe que en la mayoría de la población adulta se encuentra entre -18 y $-25 \%$. El valor promedio del strain radial en adultos es normal $>45 \%$, siendo positivo por el aumento del grosor durante la sístole. ${ }^{42}$ En el año 2013, T. Yingchoncharoen y colaboradores publicaron un metaanálisis de 24 estudios que reportó valores normales para GLS entre -15.9 y $-22.1 \%$ (promedio: $-19.7 \%$; IC 95\%: -18.9 a $-20.4 \%$ ); así mismo, investigadores del estudio Framingham reportaron valores de referencia del GLS por sexo en adultos sanos sin evidencia de enfermedades cardiovasculares de -14.4 a $-17.1 \%$ en mujeres y -14.4 a $-15.2 \%$ en hombres. ${ }^{43,44}$ Se han descrito efectos de la edad en la deformación miocárdica, pero aún sigue siendo motivo de controversia. Estudios como el de los grupos de Kuznetsova en 2008, Dalen en 2010 y Sun en $2013^{45-47}$ mostraron una reducción de los valores de strain con el avance de la edad; el STRAIN-LS fue menos negativo, el STRAIN-CS fue más negativo, mientras que el STRAIN-R se mantuvo inalterado. Fonseca y su equipo, en 2003 , utilizaron un marcador tisular con RM tridimensional comparando adultos sanos según la edad; se observaron valores de STRAIN-LS y STRAINCS menos negativos en los ancianos. ${ }^{48}$

El strain rate (SR) es la derivada temporal de la deformación y se define como el cambio en la deformación por unidad de tiempo; utiliza la unidad sec-1. Si conocemos la velocidad inicial (Vo) y la velocidad final (V1), a las cuales se deforma dicha strain longitudinal entre los dos puntos y se corrige por la longitud de la fibra: Vo-V1/Lo, se obtiene el SR. ${ }^{28}$

$$
\text { SR }=\text { Vo-V1/Lo }
$$

En cuanto al SR longitudinal en condiciones normales en un adulto, el valor promedio es 1 seg-1; el valor promedio del SR radial es 2,45 seg-1. ${ }^{42,49}$

\section{Limitaciones en la evaluación de la deformación del miocardio (STRAIN)}

Existen ciertos factores que pueden influenciar la deformación del miocardio, ya que refleja de forma directa la interacción entre la contractilidad y las condiciones de carga del miocardio. 


\section{Postcarga/precarga}

En un estudio en modelos animales ( 21 cerdos) en 2009 se demostró que el STRAIN-LS y el SR se redujeron cuando se elevó la postcarga (strain longitudinal [de $-24.2 \pm 3.2$ a $-12.1 \pm 5.5 \%, p=0.001$ ] y SR [de $-2.3 \pm 0.8$ a $-1.3 \pm 2.4 \mathrm{~s}(-1)], p=0.034)$, mientras que la precarga aumentada incrementó el STRAIN-LS y el $\mathrm{SR}$, respectivamente (de $-26.4 \pm 10.3$ a $-38.1 \pm 14.3 \%$, $\mathrm{p}=0.006)$ y SR (de $-2.3 \pm 0.9 \mathrm{a}-4.22 \pm 1.8 \mathrm{~s}[-1], \mathrm{p}=$ $0.002)$ mediante el mecanismo de Frank Starling. ${ }^{50}$ En otro estudio realizado en el 2012 en ratones se demostró que la STRAIN-R y STRAIN-CS son sensibles a los cambios en la postcarga, mientras que la SR es una medida que está menos influenciada por alteraciones en la precarga y postcarga. ${ }^{51}$

\section{Contractilidad: frecuencia cardiaca}

Se realizó un estudio en 20 cerdos que se dividieron en grupos y se sometieron a estimulación auricular (120-180/min, $n=7)$, infusión de dobutamina (DI: 2.5$20 \mu \mathrm{g} / \mathrm{kg} / \mathrm{min}, \mathrm{n}=7$ ) o infusión de esmolol con estimulación posterior (El: $0.5 \pm 0.15 \mathrm{mg} / \mathrm{kg} / \mathrm{min}$ con estimulación $120-180 / \mathrm{min}, \mathrm{n}=6$ ). Se evaluó el STRAIN, el cual correlacionó linealmente con la FEVI $(r=0.87 ; p$ $<0.0001$ ). SR pudo reflejar la función contráctil regional, mientras que el STRAIN reflejó los cambios en la geometría ventricular. En este estudio se concluyó que para la ECO de estrés cuantitativa, el SR es mejor en la cuantificación de los cambios en la función contráctil. ${ }^{52}$ En otros estudios posteriores se ha demostrado que el STRAIN también está relacionado con la frecuencia cardiaca y que el STE puede ser un método viable y útil para valoración cardiaca. ${ }^{53}$

\section{Propias de la técnica}

Este método tiene ciertas limitaciones: a pesar de que la técnica es muy precisa, no está exenta de cierta variabilidad intra-e interobservador. ${ }^{32}$ En el año 2009, Isabel Rodríguez y sus colegas realizaron un estudio para determinar valores normales de GLS y SR en 105 sujetos sanos entre 18 y 60 años; se encontraron con variabilidades del observador y entre observadores moderadas, pero susceptibles de mejora. ${ }^{54}$ Existen limitaciones intrínsecas a las imágenes, ya que requieren de buena ventana apical; se podrían utilizar imágenes tridimensionales para la valoración, pero no siempre se cuenta con ellas y tienen una resolución temporal y espacial menor. La limitación más importante en las técnicas de seguimiento es la estabilidad temporal de los patrones de seguimiento, ya que las «huellas» no son estables temporalmente (por el movimiento del plano de paso, cambios fisiológicos de las estructuras del tejido vivo y cambios de los ángulos del tejido en movimiento y el haz ultrasónico). ${ }^{36,55-57}$

\section{Aplicaciones clínicas del STRAIN en pacientes con sepsis}

En investigaciones publicadas en el año 2015 se mostró una fuerte asociación de GLS con mortalidad en pacientes con sepsis, como en el trabajo de Chang y sus colaboradores realizado en Taiwán, que fue un estudio prospectivo observacional llevado a cabo de enero de 2011 a junio de 2013 con una cohorte de 111 pacientes con diagnóstico de choque séptico. Reportaron mortalidad en la UTI de $31.5 \%$ y hospitalaria de $35.1 \%$; no hubo diferencias significativas (como en estudios previos) en la FEVI de los no sobrevivientes y los sobrevivientes. Los no sobrevivientes tuvieron GLS menos negativos que los sobrevivientes (peor función sistólica del VI), por lo que fue un adecuado predictor independiente de mortalidad en la UTI y hospitalaria (razón de riesgo $4.34, p=0.001$ y razón de riesgo 4.21, $p=0.001$, respectivamente). Tomando como punto de corte de GLS - $13 \%$, tuvo mejor sensibilidad y especificidad; área bajo la curva [AUROC]: $0.79 .{ }^{58}$

De octubre de 2012 a abril de 2015, Palmieri y su grupo realizaron en Italia un estudio prospectivo observacional en pacientes sépticos y con choque séptico, con una cohorte de 115 pacientes. A diferencia del estudio de Chang y sus colegas, se utilizó el punto de corte como normal ( $\leq-14 \%)$. Encontraron alteraciones en GLS del VI mayores en los pacientes no sobrevivientes a los siete días y 28 días (-9.1\% frente a $-10.8 \%$; $p$ $<0.05)$, a pesar de que no hubo diferencias en la FEVI entre las dos cohortes. ${ }^{59}$

En el estudio de Michael J. Lanspa en 2015 se determinó la asociación entre la $\mathrm{ScvO}_{2}$ y el STRAIN Iongitudinal anormal; lo clasificaron en mayor que $-17 \%$ como anormal y mayor que $-10 \%$ como severamente anormal. Incluyeron 42 pacientes de manera prospectiva; $60 \%$ de ellos tenían STRAIN longitudinal anormal y $16 \%$ gravemente anormal; concluyeron que el STRAIN longitudinal se asocia con baja $\mathrm{ScvO}_{2}$ (coeficiente lineal $-1.05, \mathrm{p}=0.006$, odds ratio 1.23 para $\mathrm{ScvO}_{2}<60 \%, \mathrm{p}=$ 0.016 . Los pacientes con STRAIN gravemente anormal tenían $\mathrm{ScvO}_{2}$ significativamente menor $(56.1 \%$ versus $67.5, p<0.01)$ y lactato superior $(2.7$ versus $1.9 \mathrm{mmol} /$ $\mathrm{dL}, \mathrm{p}=0.04)$. Propusieron que podría ser un sustituto no invasivo de la adecuación del DO2 durante la sepsis grave temprana o el choque séptico. ${ }^{21}$

En el año 2016 se publicó un estudio de casos y controles en la UTI en pacientes con diagnóstico de choque séptico; se les realizó ECO transtorácica a todos los pacientes dentro de las 24 horas posteriores al diagnóstico y se efectuó un nuevo ecocardiograma 
de reevaluación en el grupo de estudio en el momento de la recuperación. Se comparó este grupo con pacientes sépticos sin choque, excluyendo aquellos con otras causas de MD. El grupo de estudio tuvo un mayor grado de MD medida por GLS $(-14.5 \%$ vs $-18.3 \%, p<0.001)$, y la deformación del miocardio difirió en el diagnóstico y la recuperación $(-14.5 \%$ vs $-16.0 \%, p=0.010)$. Las mediciones ecocardiográficas convencionales como la FEVI (59\% en el grupo de estudio frente a $61 \%$ en el grupo de control, $p=0.169$ ) no difirieron entre los dos grupos. Los pacientes con choque séptico tenían más disfunción del VI, medida por GLS, que los controles emparejados con sepsis, pero sin choque. 60

Zaky y su equipo incluyeron 54 pacientes. La reducción del GLS o LVS sistólico del segmento anterior basal se asoció con mortalidad intrahospitalaria; los pacientes con TMAD reducida se vincularon con mayor riesgo de mortalidad, pero no fue estadísticamente significativo. El GLS del VI se relacionó con una menor FEVI. La reducción de TMAD global se asoció con GLS global y segmentario reducido. Concluyeron que la ECO con el método ST se puede realizar de manera factible en pacientes con sepsis pero se necesitan más estudios para evaluar la capacidad de estos nuevos índices para predecir los resultados en supervivientes y no sobrevivientes, teniendo en cuenta que los segmentos apicales se excluyeron de su análisis de deformación, por lo que la interpretación de estos resultados es algo complicada. ${ }^{61}$

De Geer y sus colaboradores, en otro estudio en el 2015, encontraron que GLS del VI es la medida más reproducible para evaluar la función cardiaca en pacientes con choque séptico, con coeficientes de correlación para la variabilidad inter e intra observador de 0.91 $(0.74-0.95, p<0.001)$ y $0.89(0.55-0.97) . p=0.002)$, respectivamente. ${ }^{62}$

Sin embargo, en términos de factibilidad para la realización de STE en pacientes sépticos, los diferentes estudios mencionados han informado tasas de exclusión por mala calidad de imagen desde 1.5 a $20 \% .{ }^{11}$

Hasta el momento existen resultados contradictorios sobre la aplicabilidad de los nuevos parámetros ecocardiográficos como el GLS o el CS del VI obtenidos mediante STE para la predicción de mortalidad a corto y largo plazo o días de estancia intrahospitalaria; estos datos han aumentado el interés en el uso de esta nueva técnica a pesar de que la asociación con la mortalidad en pacientes con sepsis también parece estar modificada por la gravedad de la enfermedad.

\section{Correlación del STRAIN en pacientes con sepsis y escalas pronósticas}

EI GLS es directamente proporcional a varios marcadores de gravedad, como el incremento en valores de
SOFA (evaluación del fallo orgánico secuencial), nivel de troponinas, elevación de péptidos natriuréticos, lactato sérico y baja de $\mathrm{ScvO}_{2}$, con independencia de los valores de FEVI. $5,57,60,63,64$

En el estudio de Chang y su grupo, en 2015, también se realizó un análisis de regresión de Cox que reveló que las puntuaciones más altas de APACHE (Acute Physiology, Age and Chronic Health Evaluation) II y GLS menos negativas eran predictores independientes de mortalidad en la UTI y el hospital. Determinó que el GLS agrega información pronóstica al APACHE II; la combinación de GLS y el puntaje APACHE II tiene un valor aditivo en la predicción de mortalidad en la UTI y hospitalaria, y el GLS puede ayudar en la identificación temprana de pacientes con choque séptico de alto riesgo en la UTI. ${ }^{58}$

En el estudio de Innocenti y sus colegas se evaluó el valor pronóstico a corto plazo del rendimiento sistólico del VI, evaluado a través de la FEVI y GLS VI en las primeras 24 horas en 147 pacientes con diagnóstico de sepsis; se realizó una estratificación de acuerdo con los terciles de SOFA evaluados en el momento del ecocardiograma (G1: puntaje de SOFA < 5; G2: puntaje de SOFA de 5-7; G3: puntaje de SOFA > 7). Se efectuó seguimiento el día siete. LVEF y GLS fueron comparables entre los tres grupos (ambos $p>0.1$ ). Se encontró que la puntuación SOFA se correlaciona bastante con mortalidad (AUC 0.635, $p=0.037$ ); una FEVI baja tiene menor correlación (AUC $0.35, p=0.022$ ) y valores menos negativos de GLS (AUC 0.73, $p=0.001$ ). También al análisis multivariado de mortalidad al día siete es mayor al tener GLS menos negativo (mejor indicativo de peor disfunción sistólica, HR 1.22/\%, $p=0.005)$ y por puntuación SOFA creciente (HR 1.22/unidad, $p=$ 0.010 ), mientras que la FEVI, ajustada por edad y puntaje de SOFA, no ingresa al modelo. La evaluación de la función sistólica del VI mediante GLS predice el pronóstico a corto plazo, independientemente de SOFA. ${ }^{65}$

\section{Usos del STRAIN en otros ámbitos clínicos}

La ECO por STE se ha aplicado cada vez más en la práctica clínica, no solo en la terapia intensiva, sino también en oncología, anestesiología, ${ }^{37}$ cardiología y neumología.

\section{MATERIAL Y MÉTODOS}

Tipo de estudio: se realizó un estudio transversal, prospectivo, de cohorte de un centro hospitalario en pacientes con sepsis ingresados en la UTI del Centro Médico ABC campus Santa Fe y campus Observatorio en la Ciudad de México, evaluados del primero de enero de 2018 al 31 de julio de 2018.

Se les realizó toma de signos vitales, laboratorios completos al momento del ingreso y a las 12 horas, así 
como toma de ecocardiograma transtorácico dentro de las primeras 24 horas del diagnóstico de sepsis.

Universo/población en estudio: todos los pacientes con diagnóstico de sepsis de acuerdo con los criterios de supervivencia de la sepsis, ingresados en UTI del Centro Médico ABC campus Santa Fe y campus Observatorio en la Ciudad de México, evaluados de enero a agosto del año 2018.

Criterios de inclusión: pacientes adultos, mayores de 18 años, con diagnóstico de sepsis. La sepsis se definió de acuerdo con los criterios de supervivencia de la sepsis 3: disfunción orgánica potencialmente mortal causada por una respuesta desregulada del huésped a la infección. Choque séptico se definió como una subcategoría de la sepsis en la que las alteraciones circulatorias y del metabolismo celular son lo suficientemente profundas como para aumentar en forma considerable la mortalidad. Criterios clínicos de sepsis: infección documentada o sospechada, escala de SOFA $>2$ puntos o qSOFA positivo (hipotensión PAS $<100 \mathrm{mmHg}$, escala de coma de Glasgow $<13$ puntos o taquipnea $>22$ respiraciones por minuto). La ecocardiografía transtorácica se realizó dentro de las primeras 24 horas de cumplir con los criterios de sepsis. Se obtuvieron imágenes en los planos transversales de los músculos papilares y apical de las cuatro cavidades con segundo armónico, con alta resolución temporal (60-100 imágenes/s) y señal de ECG de máxima definición. Las imágenes se almacenaron en formato digital para el posterior análisis off-line. Las mediciones y el análisis off-line fueron realizados por un ecocardiografista certificado perteneciente al personal del hospital. Se cuantificó la masa ventricular izquierda, los volúmenes de las cavidades, la fracción de expulsión, función diastólica y las presiones de llenado del VI de acuerdo con las últimas guías de la Sociedad Americana de Ecocardiografía, ${ }^{66}$ a través de las siguientes mediciones: volumen de la aurícula izquierda a través del método biplanar, velocidad de regurgitación tricuspídea con Doppler continuo, velocidades de la ondas s', e' y a' en la porción septal y lateral del anillo mitral con IDT, así como flujograma transmitral con Doppler pulsado para la determinación de las ondas $\mathrm{E}$ y $\mathrm{A}$. Con estas mediciones se evaluó la relación E/e' a través del promedio de la onda e' septal y e' lateral $\left(E / e^{\prime}=E /[(e ' s e p t a l+\right.$ e'lateral)/2]), así como la relación E/A. La presión sistólica de la arteria pulmonar se obtuvo mediante la sumatoria de la presión transtricuspídea y la estimación de la presión de la aurícula derecha, de acuerdo con la ecuación modificada de Bernoulli. Para la medición de la deformidad longitudinal global del VI, se adquirieron de forma bidimensional las ventanas apical cuatro cámaras, apical dos cámaras y apical tres cámaras durante tres ciclos cardiacos, para analizarse posteriormente a través del software Xcelera Phillips.
Criterios de no inclusión: pacientes con evidencia de taquiarritmias supraventriculares, embarazo, cardiopatía congénita, miocardiopatía, enfermedad valvular moderada o grave y prótesis valvular; pacientes oncológicos, quienes hubieran recibido quimioterapia, y en los que la calidad de imagen fue insuficiente.

Criterios eliminación: pacientes con calidad de imagen insuficiente o que se negaran a participar en el estudio.

Procedimiento: 1 . Se seleccionaron todos los pacientes con diagnóstico de sepsis que ingresaron al Departamento de Medicina Crítica del Centro Médico $A B C$ en el periodo del primero de enero de 2018 al 31 de julio de 2018. 2. Se obtuvieron los siguientes datos de todos los pacientes al momento del ingreso: registro, nombre, etiología de la sepsis, sexo, peso, talla, índice de masa corporal, edad, temperatura, frecuencia respiratoria, frecuencia cardiaca, tensión arterial media (TAM), ECG, inmunodepresión, hematocrito., leucocitos, $\mathrm{Na}, \mathrm{K}, \mathrm{Cr}$, lesión renal aguda, $\mathrm{pH}$, $\mathrm{FiO}_{2}, \mathrm{PaO}_{2}$, gradiente $\mathrm{A} / \mathrm{a}, \mathrm{APACHE}$ II, tipo de ingreso, diagnósticos de ingreso, cáncer, $\mathrm{VIH}$, presión sistólica, ventilación mecánica no invasiva, ventilación mecánica invasiva, nitrógeno ureico en sangre, uresis, $\mathrm{HCO}_{3}$, bilirrubinas, SAPS (Simplified Acute Physiology Score) II, requerimiento de vasopresores, dosis de los vasopresores, requerimiento de inotrópico, plaquetas, $\mathrm{PaO}_{2} / \mathrm{FiO}_{2}$, SOFA, días de estancia en la unidad, defunción, diabetes mellitus 2, hipertensión arterial sistémica, FA, tabaquismo, alcoholismo, hepatopatía, cardiopatía isquémica, enfermedad pulmonar obstructiva crónica, presión venosa arterial, hemoglobina, contenido capilar de $\mathrm{CO}_{2}$, contenido arterial de $\mathrm{O}_{2}$, contenido venoso de $\mathrm{O}_{2}$, diferencia, extracción, delta de $\mathrm{CO}_{2}$, delta/delta, lactato, lactato a las 12 horas, saturación venosa, saturación venosa las 24 horas. 3 . Se realizó el ecocardiograma durante las primeras 24 horas del diagnóstico de sepsis con un ecocardiógrafo marca Philips CX50 y un transductor de $2,5-4 \mathrm{MHz}$. Se obtuvieron imágenes en los planos transversales de los músculos papilares y apical de las cuatro cavidades con segundo armónico; las imágenes se almacenaron en formato digital para su posterior análisis off-line. Las mediciones y el análisis off-line fueron realizados por un solo ecocardiografista certificado. 4. Se cuantificó la masa ventricular izquierda, los volúmenes de las cavidades cardiacas, la fracción de expulsión, la función diastólica y las presiones de llenado del ventrículo izquierdo de acuerdo a las guías de la ASE, ${ }^{66}$ a través de las siguientes mediciones: volumen de la aurícula izquierda a través del método biplanar, velocidad de regurgitación tricuspídea con Doppler continuo, velocidades de la ondas s', e' y a' en la porción septal y lateral del anillo mitral con IDT, así como flujograma transmitral con Doppler pulsado para la determinación 
de las ondas $\mathrm{E}$ y $\mathrm{A}$. Con estas mediciones se evaluó la relación E/e' a través del promedio de la onda e' septal y e' lateral $\left(E / e^{\prime}=E /\left[\left(e^{\prime}\right.\right.\right.$ septal + e'lateral)/2]), así como la relación E/A. La presión sistólica de la arteria pulmonar se obtuvo mediante la sumatoria de la presión transtricuspídea y la estimación de la presión de la aurícula derecha, de acuerdo con la ecuación modificada de Bernoulli. 5. Para la medición de la deformidad longitudinal global del ventrículo izquierdo, se adquirieron de forma bidimensional las ventanas apical cuatro cámaras, apical dos cámaras y apical tres cámaras durante tres ciclos cardiacos para analizarse posteriormente a través del software Xcelera Phillips. 6. Se registró lo siguiente en la base de datos: LVEDV (volumen del ventrículo izquierdo al final de la diástole), LVEF, S'med, e'med, a'med, s'lat, e'lat, a'lat, E, A, E/A, E/a', FACVD (fracción de acortamiento del ventrículo derecho), TAPSE, disfunción diastólica, S'VD, GLS, strain circunferencial, TIV, disfunción diastólica (DD), PP, DS, MVI, GPR, GIT, PSAP, Vmax IT, VAI (volumen aurícula izquierda). 7. Todos los pacientes ingresados fueron manejados mediante reanimación temprana, manteniendo los siguientes objetivos: a. PVC 0-5 mmHg. b. TAM $>65 \mathrm{mmHg}$. c. Uresis $>0.5$ $\mathrm{mL} / \mathrm{g} / \mathrm{h}$. d. $\mathrm{ScvO}_{2}>70 \%$. e. Depuración de lactato. 8 . Se realizó el análisis de las variables.

Análisis estadístico: Se utilizó estadística descriptiva para las variables numéricas, que incluyeron medidas de tendencia central y dispersión, mediana y rangos intercuartilares (RIQ). Las variables categóricas fueron representadas como frecuencias absolutas y relativas. La prueba de hipótesis para variables lineales fue $U$ de Mann-Whitney; para variables categóricas se hizo prueba de $\chi^{2}$ o prueba exacta de Fisher si la frecuencia fue igual o menor a 5. Se realizó análisis de sensibilidad y especificidad, así como representación gráfica usando curvas ROC para encontrar puntos de corte capaces de discriminar mortalidad. Los puntajes de variables y sus ítems fueron incluidos en la construcción de modelos de regresión logística y fueron representados como razón de momios con intervalos de confianza de $95 \%$. Se buscó la correlación entre variables hemodinámicas (PVC y TAM) con el GLS y CS utilizando la r de Pearson. Todas las pruebas de hipótesis tuvieron un error alfa ajustado para una $p<0.05$. La paquetería estadística utilizada fue STATA SE (Data Analysis and Statistical Software) 13.1 y el software SPSS (Statistical Package for the Social Science).

Implicaciones éticas: El presente estudio cumple los lineamientos mencionados en: El Comité de Ética e Investigación del Centro Médico ABC. La Declaración de Helsinki. La Ley General de Salud. El Reglamento de la Ley General en Materia de Investigación en Salud, donde se considera este tipo de estudios como investigación sin riesgo; requiere de consentimiento por parte del paciente o familiares al ingreso a terapia intensiva. Se trató de una muestra confidencial.

\section{RESULTADOS}

\section{Características generales}

Se incluyeron datos de 30 pacientes, 17 del sexo masculino (56.7\%), con mediana de edad de 68.5 años (RIQ 56-84) y una mediana de estancia intrahospitalaria de 12 días (RIQ 7-17). La etiología de la sepsis en orden de frecuencia fue pulmonar, en 15 pacientes (50\%); abdominal, en $12(40 \%)$ y urinario, en tres (10\%). Del total, siete pacientes fallecieron (tasa de defunción de 23.3\%). Entre ellos, la puntuación media de APACHE II fue de 16.1 puntos, SAPS fue de 30 puntos y SOFA de 9.3 puntos.

La duración media de estancia en la UCI fue de 19.1 días (3-185 días).

Valorado de manera independiente por CS, se encontró que $36 \%$ de la población presentó disfunción sistólica del VI, mientras que valorado por GLS, se obtuvo $40 \%$ de la población.

Los pacientes evaluados se clasificaron en dos grupos: los no sobrevivientes y los sobrevivientes. Al comparar los grupos, no hubo diferencias demográficas significativas; se observó una diferencia de proporciones en el número de pacientes con diabetes, pero el resto de las características generales fue similar entre los grupos (Tabla 1).

En comparación con los sobrevivientes, los que no sobrevivieron fueron en su mayoría ingresos de tipo médico, con sepsis de etiología respiratoria o abdominal, y tuvieron puntajes SAPS II arriba de 42.

\section{Mediciones ecocardiográficas}

Al hacer la revisión de las medidas ecocardiográficas en pacientes con sepsis que sobrevivieron contra los no sobrevivientes, se observaron diferencias en las medianas de PSAP, VAI, CS y GLS.

No hubo diferencias significativas en la FEVI, LVEDV, E, A, E/A, E/e, VAl o la FACVD (Tabla 2, Figuras 1A y 1B).

Se encontró un paciente con FEVI reducida $(<50 \%)$ en el grupo de los no sobrevivientes, el cual exhibió GLS y CS menos negativos. Por el contrario, entre los pacientes con FEVI preservada (>50\%), los no sobrevivientes exhibieron GLS y CS reducidos. Entre el grupo de pacientes con FEVI conservada, cinco pacientes tenían LV hipercinéticos (FEVI mayor que $70 \%$ ). Sin embargo, el número limitado de pacientes puede conducir a diferencias no significativas entre los sobrevivientes y los no sobrevivientes.

Existen algunas variables hemodinámicas, como la PVC, la Fc y la TAM, que pueden alterar la función sis- 
tólica del VI. En este estudio se evaluaron los pacientes ya reanimados con TAM $>70 \mathrm{mmHg}$, sin evidencia de taquicardia al momento del ecocardiograma. Se realizó una correlación entre la variables hemodinámicas al ingreso y las medidas ecocardiográficas; se encontró una correlación positiva entre la PVC de ingreso y el GLS [PVC y GLS $r=0.408, p=0.027$; PVC y CS $r=0.213$, $p$ $=0.307$. TAM y GLS $r=-0.251, p=0.1818$; TAM y CS $r=-0.198, p=0.341]$.

\section{Análisis de curva ROC}

Al probar la capacidad para discriminar sobrevivientes contra no sobrevivientes, se observó que el CS fue marginalmente superior que el GLS, APACHE II, SAPS II y SOFA (Figura 2A). Al probar la capacidad de CS y GLS para discriminar estancia hospitalaria mayor a siete días, se observó que las medidas ecocardiográficas fueron superiores (Figura 2B).

Tabla 1: Características basales de los pacientes con sepsis admitidos en la Unidad de Terapia Intensiva (UTI).

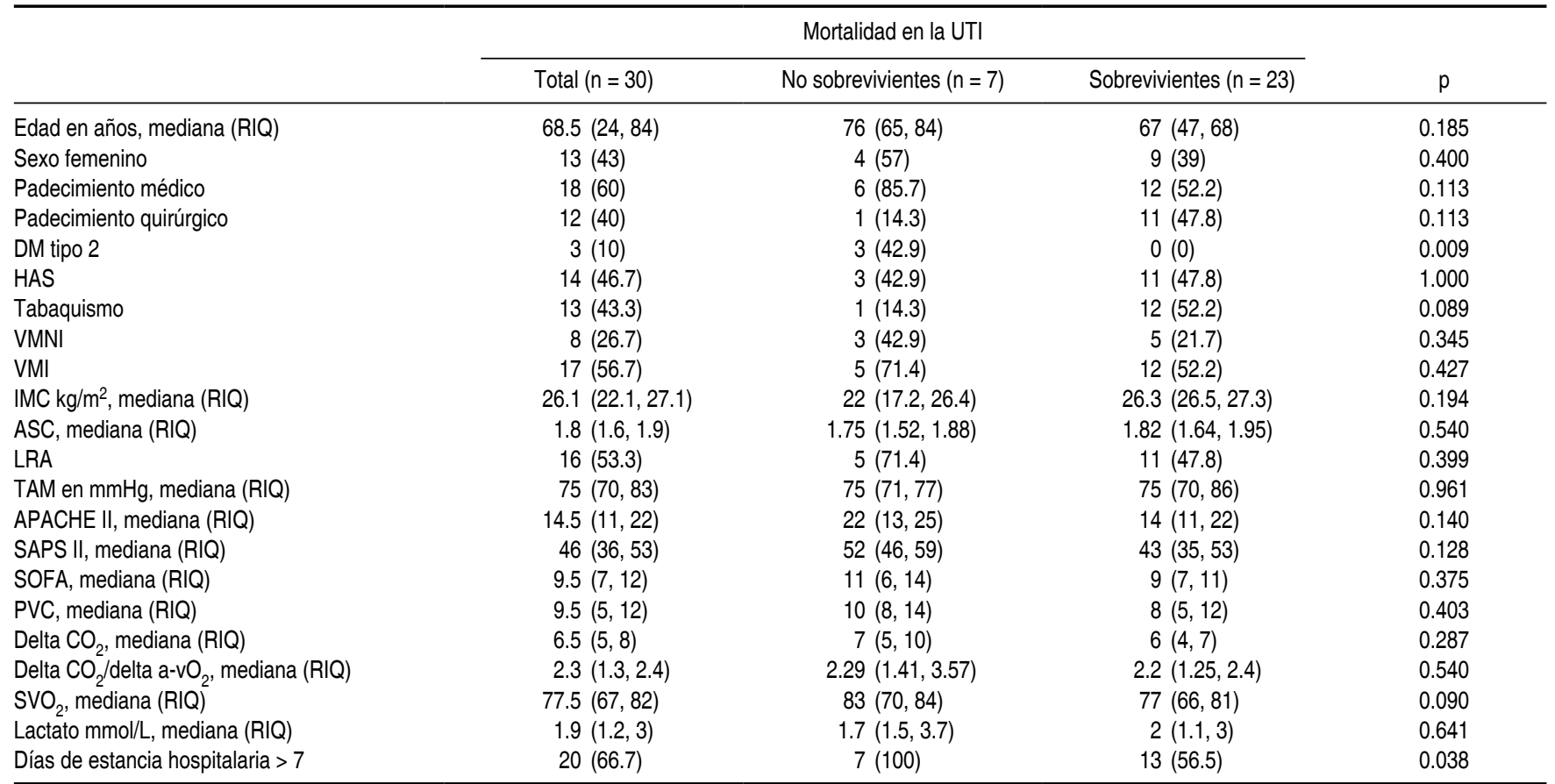

Datos como $\mathrm{n}(\%)$, a menos que se especifique de otra manera.

Abreviaturas: $\mathrm{DM}=$ diabetes mellitus, $\mathrm{HAS}=$ hipertensión arterial sistémica, $\mathrm{VMNI}=$ ventilación mecánica no invasiva, $\mathrm{VMI}=$ ventilación mecánica invasiva, IMC = índice de masa corporal, ASC = área de superficie corporal, LRA = lesión renal aguda, TAM = tensión arterial media, APACHE II = Acute Physiology Age and Chronic Health Evaluation, SAPS II = Simplified Acute Physiology Score, SOFA = Sequential Organ Failure Assessment, PVC = presión venosa central, delta $\mathrm{CO}_{2}=$ dióxido de carbono, delta a- $\mathrm{vO}_{2}=$ diferencia de oxígeno arteriovenoso, $\mathrm{ScvO}_{2}=$ saturación venosa de oxígeno central.

Tabla 2: Características ecocardiográficas de los pacientes con sepsis evaluados en la Unidad de Terapia Intensiva.

\begin{tabular}{|c|c|c|c|c|}
\hline & Total $(n=30)$ & No sobrevivientes $(n=7)$ & Sobrevivientes $(n=23)$ & $\mathrm{p}$ \\
\hline PSAP (mmHg), mediana (RIQ) & $43(34,51)$ & $50(44,63)$ & $39(34,47)$ & 0.042 \\
\hline LVEDV (mL), mediana (RIQ) & $44(38,60)$ & $45(30,68)$ & $43(38,60)$ & 0.922 \\
\hline $\mathrm{E}(\mathrm{cm} / \mathrm{s})$, mediana $(\mathrm{RIQ})$ & $76.1(64.5,97.8)$ & $67.8(63.4,94.3)$ & $85(70.8,99.3)$ & 0.476 \\
\hline $\mathrm{E} / \mathrm{A}$, mediana $(\mathrm{R} \mid \mathrm{Q})$ & $1.0(0.7,1.4)$ & $0.76(0.7,1.0)$ & $1.1(0.7,1.4)$ & 0.229 \\
\hline E/e', mediana (RIQ) & $9.3(7,9.3)$ & $10.8(6.6,13.6)$ & $9.2(7.0,11.9)$ & 0.600 \\
\hline$A(\mathrm{~cm} / \mathrm{s})$ & $84(58.8,100)$ & $88(61.9,99.3)$ & $76.8(56,102)$ & 0.641 \\
\hline CS (\%), mediana (RIQ) & $-17(-24.7,-13.9)$ & $-13.1(-17.6,-12.5)$ & $-20.5(-26.1,-15.6)$ & 0.029 \\
\hline GLS (\%), mediana (RIQ) & $-17.5(-20.2,-14.3)$ & $-14.3(-15.4,-12.7)$ & $-18(-20.4,-14.9)$ & 0.016 \\
\hline FACVD (\%) & $54(45,56)$ & $50(40,56)$ & $55(45,60)$ & 0.375 \\
\hline
\end{tabular}

Abreviaturas: FEVI $(\%)$ = fracción de eyección del ventrículo izquierdo, PSAP $(\mathrm{mmHg})=$ presión sistólica de la arteria pulmonar, DD $(\%)=$ disfunción diastólica, LVEDV $(\mathrm{mL})=$ volumen del ventrículo izquierdo al final de la diástole, $\mathrm{E}(\mathrm{cm} / \mathrm{s})=\|$ lenado diastólico precoz, $\mathrm{A}(\mathrm{cm} / \mathrm{s})=$ contracción auricular, $E / A=$ relación de llenado diastólico precoz y la contracción auricular, $\mathrm{E} / \mathrm{e}=$ velocidad transmitral temprana a la relación de la velocidad diastólica precoz anular mediante IDT del tejido, VAI $=$ volumen aurícula izquierda, CS = strain circunferencial, GLS = strain global longitudinal, FACVD = fracción de acortamiento del ventrículo derecho. 


\section{Capacidad de predicción de los puntos de corte}

Se seleccionaron puntos de corte para la discriminación de supervivientes contra no sobrevivientes mediante el análisis de sensibilidad y especificidad para las siguientes medidas ecocardiográficas:

$$
\begin{aligned}
& \text { - CS } \geq-15.1 \text { (S: } 71.43 \%, E: 83.33 \% \text {, } \\
& \text { LR(+) 4.3, LR(-) 0.34) y } \\
& \text { - GLS } \geq-15.4 \text { (S: 85.71\%, E: 73.91\%, } \\
& \operatorname{LR}(+) \text { 3.3, LR(-) 0.19). }
\end{aligned}
$$

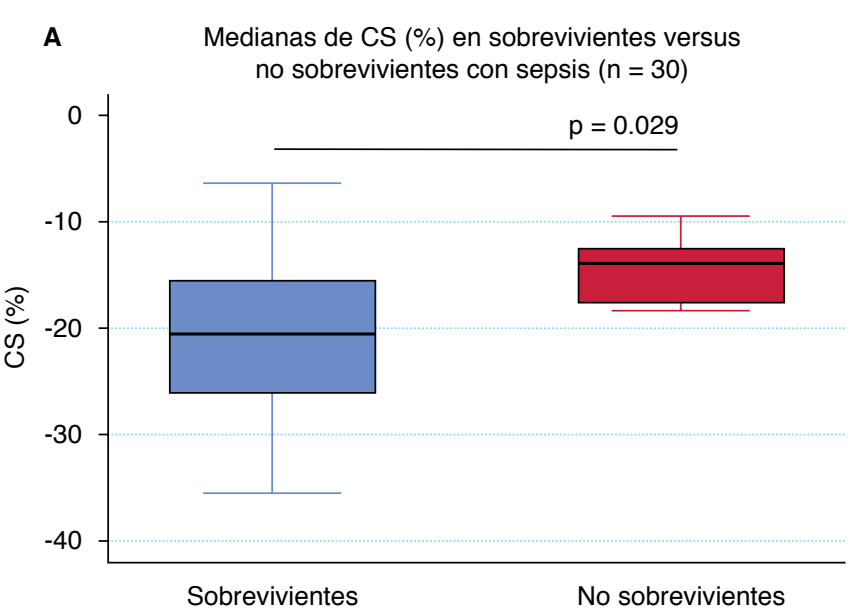

\section{Análisis multivariado}

En el análisis multivariado se encontró que un CS $\geq$ -15.1 fue predictivo de mortalidad durante la hospitalización en el periodo y población de estudio, ajustado para otras variables ecocardiográficas como FEVI $<55 \%$, GLS $\geq-15.4$ y confusores como sexo [RM = 10.23 (IC 95\%, 1.01-103.2), $\mathrm{p}<0.049]$.

En modelos de regresión lineal, no se encontraron variables ecocardiográficas predictoras de días de estancia hospitalaria en el periodo y población de estudio.

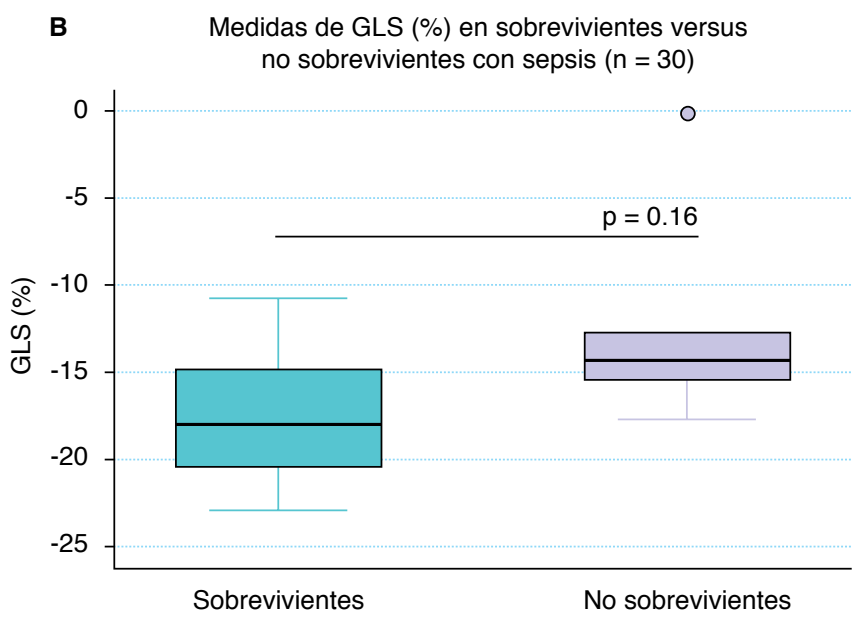

Abreviaturas: $\mathrm{CS}=$ strain circunferencial, GLS = strain global longitudinal.

Figura 1: A. Medianas de los valores de CS y GLS (\%) en pacientes no sobrevivientes versus sobrevivientes con sepsis. B. Curva ROC que muestran la capacidad de discriminar pacientes sobrevivientes versus no sobrevivientes usando medidas ecocardiográficas y escalas de predicción para mortalidad en pacientes con sepsis ingresados a la Unidad de Terapia Intensiva.

A

Curva ROC para discriminación de sobrevivientes versus no sobrevivientes en pacientes con sepsis ingresados a la UTI.
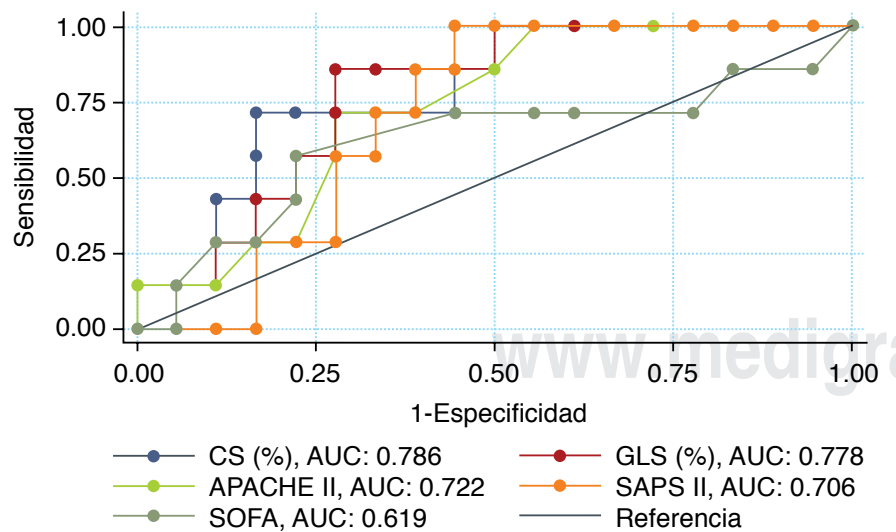

B Curva ROC para discriminación de estancia mayor a siete días en pacientes con sepsis ingresados a la UTI.

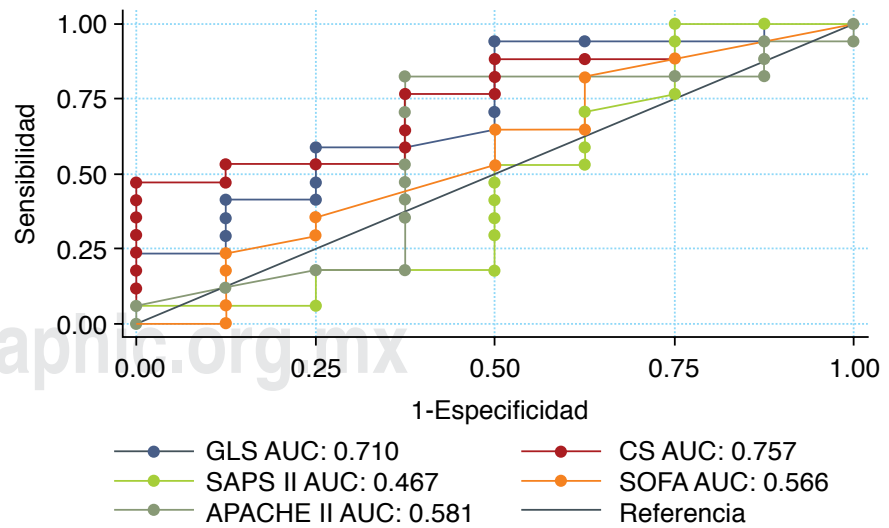

Abreviaturas: $\mathrm{CS}=$ strain circunferencial, GLS = strain global longitudinal, APACHE II = Acute Physiology, Age and Chronic Health Evaluation, SAPS II = Simplified Acute Physiology Score, SOFA = Sequential Organ Failure Assessment.

Figura 2: A. Curva ROC que muestran la capacidad de discriminar pacientes sobrevivientes versus no sobrevivientes usando medidas ecocardiográficas y escalas de predicción para mortalidad en pacientes con sepsis ingresados a la Unidad de Terapia Intensiva. B. Curva ROC que muestra la capacidad de discriminar una estancia hospitalaria mayor a siete días usando medidas ecocardiográficas y escalas de predicción para mortalidad en pacientes con sepsis ingresados a la Unidad de Terapia Intensiva. 


\section{Limitaciones del estudio}

En primer lugar, fue un número pequeño de pacientes, en un solo centro hospitalario (de dos sedes); los hallazgos carecen de verificación externa. Por lo tanto, el valor pronóstico de GLS y CS en pacientes sépticos requiere una mayor confirmación en estudios multicéntricos a gran escala. En segundo lugar, todos los pacientes del estudio ingresaron en la UTI con diagnóstico de sepsis, pero con enfermedades subyacentes y gravedad diferente, con variación en el tratamiento (ventilación mecánica, vasopresores e inotrópicos); el ser un grupo heterogéneo pudo repercutir en el pronóstico. En tercer lugar, hubo factores derivados de la propia ecocardiografía que pudieron influir, tales como la calidad de la imagen - que, a su vez, pudo ser alterada por ventanas acústicas deficientes y artefactos de la VM-, el decúbito lateral izquierdo (que es la posición ideal para un estudio ecocardiográfico adecuado) puede ser limitado en pacientes críticos, los valores de referencia de GLS y CS no están estandarizados en los proveedores de ultrasonido, por lo que pueden variar dependiendo el algoritmo. En cuarto lugar, los rangos de referencia en otros estudios se obtuvieron derivados de pacientes estables y aún existe discrepancia en cuanto al rango de valores en adultos. En quinto lugar, no se realizaron ecocardiogramas de seguimiento en los pacientes, con lo que valoraríamos los efectos de las intervenciones médicas y la progresión clínica de la sepsis, cosa que no era como tal el objetivo del estudio.

\section{DISCUSIÓN}

Es el primer estudio en México que valora la utilidad de los nuevos parámetros ecocardiográficos como el GLS y CS como indicadores pronósticos en pacientes con sepsis en la UTI, así como su aplicación concomitante con escalas pronósticas como el APACHE II, que puede favorecer una mejor evaluación del riesgo del paciente séptico.

La incidencia de la disfunción sistólica del VI valorada por $C S \geq-15.1$ y GLS $\geq-15.4$ en los pacientes sépticos de la UTI fue de $43 \%$, ligeramente mayor de lo esperado según la incidencia registrada por Blanco J. Muriel y su colaboradores, de al menos un tercio de los pacientes con sepsis. ${ }^{16}$ Valorado de manera independiente por CS, se encontró que $36 \%$ de la población presentó disfunción sistólica del VI, mientras que valorado por GLS, se obtuvo $40 \%$ de la población. Este fenómeno puede estar explicado por varios factores como la inflamación, la producción de radicales libres, las toxinas y la disfunción mitocondrial. ${ }^{5}$ Se obtuvo en este estudio una tasa de defunción de $23.3 \%$, similar a la registrada en la literatura por Vincent y su grupo desde 2006 o Vallabhajosyula y sus colegas este año, ${ }^{1}$ lo que remarca que la detección temprana de la MD en pacientes sépticos puede ser crucial para predecir el pronóstico y facilitar el manejo en etapas tempranas.

Aunque una mayor proporción de no sobrevivientes tenía diabetes mellitus tipo 2 ( $p=0.009$ ) (Tabla 1), el tiempo de diagnóstico de la misma, el apego al tratamiento y los controles variaron en nuestros pacientes, siendo difícil evaluar con precisión el impacto en la función sistólica de los pacientes diabéticos inscritos en la UCl.

Se identificó un valor de corte de CS $\geq-15.1$ (S: $71.43 \%$, E: $83.33 \%, \mathrm{LR}(+)$ 4.3, LR(-) 0.34$)$ y GLS $\geq$ -15.4 (S:85.71\%, E: 73.91\%, LR(+) 3.3, LR(-) 0.19) con óptima sensibilidad y especificidad, a diferencia de otros estudios en los que se utilizaron valores de corte de GLS desde -10 a $-18 \% .{ }^{64,66,67}$

Los estudios clínicos publicados en fechas recientes que valoran el papel del STE en la evaluación del VI en la sepsis y choque séptico han arrojado resultados contradictorios sobre la mortalidad a corto y largo plazo. Existen tres estudios prospectivos observacionales relevantes desde 2015. El primero fue realizado en Taiwán por Chang y su equipo; incluyeron 111 pacientes con diagnóstico de choque séptico; los no sobrevivientes tuvieron GLS menos negativos que los sobrevivientes, lo que reflejaba peor función sistólica del VI; fue un adecuado predictor independiente de mortalidad en la $\mathrm{UCl}$ y hospitalaria (razón de riesgo 4.34, $p=0.001$ y razón de riesgo 4.21, $p=0.001$, respectivamente). Tomando como punto de corte de GLS -13\%, tuvo mejor sensibilidad y especificidad, área bajo la curva [AUROC]: $0.79 .{ }^{58} \mathrm{El}$ segundo estudio, por Palmieri y sus colaboradores, incluyó 115 pacientes sépticos y con choque séptico; utilizaron el punto de corte como normal $(\leq-14 \%)$; encontraron alteraciones en GLS del VI mayores en los pacientes no sobrevivientes a los siete días y 28 días $(-9.1 \%$ frente a $-10.8 \% ; p<0.05)$, a pesar de que no hubo diferencias en la FEVI entre las dos cohortes. ${ }^{59}$ El tercer estudio, dirigido por Michael J. Lanspa y su grupo, determinó la asociación entre la $\mathrm{ScvO}_{2}$ y el STRAIN longitudinal anormal; incluyeron 42 pacientes y concluyeron que el GLS se asocia con baja $\mathrm{ScvO}_{2}$ (coeficiente lineal -1.05, $\mathrm{p}=$ 0.006 , odds ratio 1.23 para $\left.\mathrm{ScvO}_{2}<60 \%, p=0.016\right)$; tenían $\mathrm{ScvO}_{2}$ significativamente menor $(56.1 \%$ versus $67.5 \%, p<0.01)$ y lactato superior $(2.7$ versus $1.9 \mathrm{mmol} /$ $\mathrm{dL}, \mathrm{p}=0.04$ ), por lo que propusieron que podría ser un sustituto no invasivo de la adecuación del $\mathrm{DO}_{2}$ durante la sepsis grave temprana o el choque séptico. ${ }^{21}$

En este estudio se demostró que el GLS y el CS pueden servir como marcadores pronósticos que podrían agregar información incremental de los pacientes con sepsis en la UTI, ya que representan la función sistólica del VI. Se incluyeron 30 pacientes, $56.7 \%$ del sexo masculino (17), con mediana de edad de 68.5 años (RIQ 56-84); la mediana de estancia intrahospitalaria fue de 12 días (RIQ 7-17). La etiología del sitio de sepsis fue pulmonar en $50 \%$ de los pacientes (15), abdominal en 
$40 \%$ (12) y urinaria en $10 \%$ (3). Se observaron diferencias en las medianas de PSAP, VAI, CS y GLS al revisar las medidas ecocardiográficas en pacientes con sepsis que sobrevivieron contra los no sobrevivientes. El CS fue marginalmente superior en cuanto a capacidad para discriminar sobrevivientes contra no sobrevivientes, en comparación con GLS, APACHE II, SAPS II y SOFA (Figura 2). Al probar la capacidad de CS y GLS para discriminar estancia hospitalaria mayor a siete días, se observó que las medidas ecocardiográficas fueron superiores. En el análisis multivariado se encontró que valores de $C S \geq-15.1$ fueron predictivos de mortalidad, ajustado para otras variables ecocardiográficas como $\mathrm{FEVI}<55 \%$, GLS $\geq-15.4$ y confusores como sexo [RM $=10.23(\mathrm{IC} 95 \%, 1.01-103.2), \mathrm{p}<0.049]$. En cuanto a los modelos de regresión lineal, no se encontraron variables ecocardiográficas predictoras de días de estancia hospitalaria en el periodo y población de estudio. Evaluamos solo la función del VI, considerando que el VD se ve fácilmente afectado por la precarga, arritmias, hipoxia y el modo ventilatorio. Sin embargo también existen algunas variables hemodinámicas, como la PVC y la TAM, que pueden alterar la función sistólica del VI. En el año 2009, Rosner y sus colegas realizaron un estudio en 21 puercos; demostraron que el GLS y la SR se redujeron cuando aumentó la postcarga GLS (de $-24.2 \pm 3.2$ a $-12.1 \pm$ $5.5 \%, p=0.001)$ y SR (de $-2.3 \pm 0.8 \mathrm{a}-1.3 \pm 2.4 \mathrm{~s}(-1), \mathrm{p}$ $=0.034)$, mientras que la precarga incrementada aumentó el strain longitudinal de $-26.4 \pm 10.3$ a $-38.1 \pm 14.3 \%$, $\mathrm{p}=0.006$ y la SR de $-2.3 \pm 0.9$ a $-4.22 \pm 1.8 \mathrm{~s}(-1), \mathrm{p}=$ 0.002 mediante el mecanismo de Frank Starling. ${ }^{50}$ En otro estudio, realizado por Ferferieva y su equipo en 2012 en ratones, se demostró que el SR es una medida que está menos influenciada por alteraciones en la precarga y postcarga. ${ }^{51}$ En este trabajo se analizó la correlación entre la variables hemodinámicas al ingreso y medidas ecocardiográficas; se encontró una correlación positiva entre la PVC de ingreso y el GLS [PVC y GLS r $=0.408, p=0.027 ;$ PVC y CS $r=0.213, p=0.307$. TAM y GLS $r=-0.251, p=0.1818$; TAM y CS $r=-0.198, p=$ 0.341 ], lo cual no fue así para el CS. De los 30 pacientes incluidos, solo ocho presentaron PVC $<5 \mathrm{mmHg}$, por lo que es probable que los valores de GLS fueran más negativos por el mecanismo de Frank Starling, lo cual no afecta con tal magnitud la medición del CS, siendo un valor más confiable para pacientes críticos.

Desde hace varios años, se sabe que la FEVI no refleja de manera adecuada la función sistólica, sobre todo al inicio de la patología. EI STE anormal tiene el potencial de identificar pacientes con FEVI normal que tienen un mayor riesgo de eventos adversos, lo que hace del STE una medida más sensible de SCM clínicamente relevante. Innocenti y sus colaboradores demostraron en este año que la FEVI baja tiene menor correlación (AUC 0.35, $p=0.022$ ) que valores menos negativos de GLS (AUC
$0.73, p=0.001)$ para mortalidad en pacientes sépticos, ${ }^{65}$ lo que coincide con nuestro grupo de los no sobrevivientes, ya que solo un paciente presentó FEVI reducida.

El estudio de Chang y su grupo en 2015 determinó que el GLS agrega información pronóstica al APACHE Il y la combinación tiene un valor aditivo en la predicción de mortalidad en la UCl y hospitalaria. ${ }^{58}$ En el estudio de Innocenti y sus colegas, se evaluaron 147 pacientes con diagnóstico de sepsis y se encontró que la puntuación SOFA correlaciona bastante con mortalidad (AUC 0.635, p = 0.037); sin embargo, los valores menos negativos de GLS fueron los que obtuvieron mejor correlación (AUC 0.73, $p=0.001$ ); también se realizó un análisis multivariado de mortalidad al día siete, el cual fue mayor al tener GLS menos negativo (mejor indicativo de peor disfunción sistólica, HR 1.22/\%, p = 0.005) y por puntuación SOFA creciente (HR 1.22/unidad, $p=$ $0.010) .{ }^{65}$ En este estudio también evaluamos las relaciones potenciales de las escalas pronósticas con GLS y CS, pero no se encontró una asociación significativa entre los mismos, lo cual pudo estar ocasionado por los puntajes obtenidos relativamente bajos (APACHE II fue de 16.1 puntos, SAPS fue de 30 puntos y SOFA de 9.3 puntos) o el escaso número de pacientes.

\section{CONCLUSIONES}

El desarrollo de nuevas técnicas de ECO, como el STE, ha facilitado la capacidad de evaluar la función del VI mediante la evaluación cuantitativa de la deformación miocárdica. Aunque son técnicas complejas, si se realizan e interpretan de forma apropiada, son de gran utilidad en diferentes condiciones clínicas. La MD puede ser un marcador temprano de mortalidad en pacientes con sepsis; obtenida a través de la medición del GLS y CS, podría ser un predictor pronóstico confiable de los resultados de los pacientes en UTI, pudiendo también potencializar escalas como el APACHE II, SOFA y SAPS II para permitir la identificación temprana de pacientes sépticos de alto riesgo. Aún existen ciertos obstáculos para la aplicación clínica regular de GLS y CS en pacientes sépticos en UTI. A pesar de ser un estudio a la cabecera del paciente, hay que tomar en cuenta la gravedad propia de la enfermedad y las comorbilidades previas, y debe limitarse a un específico grupo de pacientes (ya que existen factores que pueden modificar sus resultados y pacientes con uso de quimioterapia, valvulopatías, FA, etcétera deben ser excluidos). El límite de GLS óptimo para la predicción de la mortalidad en estos pacientes sigue siendo incierto y las diferencias intrínsecas entre las poblaciones podrían contribuir a las diferencias observadas; sin embargo, se sabe que es un parámetro eficaz para la cuantificación de la función ventricular izquierda, más sensible incluso que la FEVI por ECO bidimensional, depende relativamente menos del operador y las condiciones de carga. 
El strain más utilizado en investigación es el GLS, ya que brinda información completa sobre los 17 segmentos del VI, así como sobre su función global. La naturaleza reversible de la MD en la sepsis también fue demostrable. Esta técnica ecocardiográfica es útil en el diagnóstico y control de la disfunción. Sin embargo, los resultados actuales deben confirmarse en estudios adicionales a gran escala y multicéntricos. Por ello, aún permanece en el campo de la investigación clínica para los pacientes en estado crítico. Es necesario adoptar protocolos de estudio más uniformes entre los grupos de pacientes sépticos para realizar una investigación adecuada, hacer estudios multicéntricos, de seguimiento mediante ECO y con mayor número de pacientes para lograr un adecuado plan de tratamiento temprano.

\section{BIBLIOGRAFÍA}

1. Vincent JL, Sakr Y, Sprung CL, Ranieri VM, Reinhart K, Gerlach $\mathrm{H}$, et al. Sepsis in European intensive care units: results of the SOAP study. Crit Care Med. 2006;34(2):344-353.

2. Vallabhajosyula S, Jentzer JC, Geske JB, Kumar M, Sakhuja A, Singhal A, et al. New-onset heart failure and mortality in hospital survivors of sepsis-related left ventricular dysfunction. choque. 2018;49(2):144-149.

3. Russell JA, Boyd J, Nakada T, Thair S, Walley KR. Molecular mechanisms of sepsis. Contrib Microbiol. 2011;17:48-85.

4. Conway-Morris A, Wilson J, Shankar-Hari M. Immune activation in sepsis. Crit Care Clin. 2018;34(1):29-42.

5. Kakihana Y, Ito T, Nakahara M, Yamaguchi K, Yasuda T. Sepsis-induced myocardial dysfunction: pathophysiology and management. J Intensive Care. 2016;4:22.

6. Bruni FD, Komwatana P, Soulsby ME, Hess ML. Endotoxin and myocardial failure: role of the myofibril and venous return. $\mathrm{Am} \mathrm{J}$ Phys. 1978;235(2):H150-H156.

7. Cunnion RE, Schaer GL, Parker MM, Natanson C, Parrillo JE. The coronary circulation in human septic shock. Circulation. 1986;73(4):637-644.

8. Sato R, Nasu M. A review of sepsis-induced cardiomyopathy. $J$ Intensive Care. 2015;3:48.

9. Madorin WS, Rui T, Sugimoto N, Handa O, Cepinskas G, Kvietys PR. Cardiac myocytes activated by septic plasma promote neutrophil transendothelial migration: role of plateletactivating factor and the chemokines LIX and KC. Circ Res. 2004;94(7):944-951.

10. Rudiger A, Singer M. Mechanisms of sepsis-induced cardiac dysfunction. Crit Care Med. 2007;35(6):1599-1608.

11. Ehrman RR, Sullivan AN, Favot MJ, Sherwin RL, Reynolds CA, Abidov A, et al. Pathophysiology, echocardiographic evaluation, biomarker findings, and prognostic implications of septic cardiomyopathy: a review of the literature. Crit Care. 2018;22(1):112.

12. Bednarczyk JM, Fridfinnson JA, Kumar A, Blanchard L, Rabbani $\mathrm{R}$, Bell $\mathrm{D}$, et al. Incorporating dynamic assessment of fluid responsiveness into goal-directed therapy: a systematic review and meta-analysis. Crit Care Med. 2017;45(9):1538-1545.

13. Cherpanath TG, Hirsch A, Geerts BF, Lagrand WK, Leeflang $\mathrm{MM}$, Schultz MJ, et al. Predicting fluid responsiveness by passive leg raising: a systematic review and meta-analysis of 23 clinical trials. Crit Care Med. 2016;44(5):981-991.

14. Ma IWY, Caplin JD, Azad A, Wilson C, Fifer MA, Bagchi A, et al. Correlation of carotid blood flow and corrected carotid flow time with invasive cardiac output measurements. Crit Ultrasound $J$. 2017;9(1):10.

15. Jardin F, Fourme T, Page B, Loubieres Y, Vieillard-Baron A, Beauchet $A$, et al. Persistent preload defect in severe sepsis despite fluid loading: A longitudinal echocardiographic study in patients with septic shock. Chest. 1999;116(5):1354-1359.
16. Blanco J, Muriel-Bombín A, Sagredo V, Taboada F, Gandía $F$, Tamayo L, et al. Incidence, organ dysfunction and mortality in severe sepsis: a Spanish multicentre study. Crit Care. 2008;12(6):R158.

17. Calvin JE, Driedger AA, Sibbad WJ. Una evaluación de la función del miocardio en la sepsis humana mediante gammagrafía cardiaca controlada. Chest. 1981;80:579-586.

18. Parker MM, Shelhamer JH, Bacharach SL, Green MV, Natanson $\mathrm{C}$, Frederick TM, et al. Profound but reversible myocardial depression in patients with septic shock. Ann Intern Med. 1984;100(4):483-490.

19. Vallabhajosyula S, Pruthi S, Shah S, Wiley BM, Mankad SV, Jentzer JC. Basic and advanced echocardiographic evaluation of myocardial dysfunction in sepsis and septic shock. Anaesth Intensive Care. 2018;46(1):13-24.

20. Levy RJ, Piel DA, Acton PD, Zhou R, Ferrari VA, Karp JS, et al. Evidence of myocardial hibernation in the septic heart. Crit Care Med. 2005;33(12):2752-2756.

21. Lanspa MJ, Pittman JE, Hirshberg EL, Wilson EL, Olsen T, Brown $\mathrm{SM}$, et al. Association of left ventricular longitudinal strain with central venous oxygen saturation and serum lactate in patients with early severe sepsis and septic shock. Crit Care. 2015;19:304.

22. Del Castillo JM, Herszkowicz N, Ferreira C. Speckle tracking-A contratilidade miocárdica em sintonia fina. Rev Bras Ecocardiogr Imagem Cardiovasc. 2010;23(3):46-54.

23. Lanspa MJ, Gutsche AR, Wilson EL, Olsen TD, Hirshberg EL, Knox DB, et al. Application of a simplified definition of diastolic function in severe sepsis and septic shock. Crit Care. 2016;20(1):243.

24. Sevilla Berrios RA, O'Horo JC, Velagapudi V, Pulido JN. Correlation of left ventricular systolic dysfunction determined by low ejection fraction and 30-day mortality in patients with severe sepsis and septic shock: a systematic review and meta-analysis. J Crit Care. 2014;29(4):495-499.

25. Boissier F, Razazi K, Seemann A, Bedet A, Thille AW, de Prost $\mathrm{N}$, et al. Left ventricular systolic dysfunction during septic shock: the role of loading conditions. Intensive Care Med. 2017;43(5):633-642.

26. Cosín-Aguilar J, Hernándiz-Martínez A. La disposición de las fibras miocárdicas en una banda condiciona la morfología y la función del corazón. Rev Esp Cardiol. 2013;66(10):768-700.

27. Gorcsan J 3rd, Tanaka H. Echocardiographic assessment of myocardial strain. J Am Coll Cardiol. 2011;58(14):1401-1413.

28. Holly G, Giuseppe C, Haruhiko A, Susan W, Scipione C, Federico $\mathrm{G}$, et al. Assessment of myocardial mechanics using speckle tracking echocardiography: fundamentals and clinical applications. J Am Soc Echocardiogr. 2010;23:351-369.

29. Dandel M, Lehmkuhl, Knosalla C, Suramelashvili N, Hetzer R. Strain and strain rate imaging by echocardiography. Basic concepts and clinical applicability. Curr Cardiol Rev. 2009;5:133-148.

30. Amundsen BH, Helle-Valle T, Edvardsen T, Torp H, Crosby J, Lyseggen $\mathrm{E}$, et al. Noninvasive myocardial strain measurement by speckle tracking echocardiography: validation against sonomicrometry and tagged magnetic resonance imaging. $J \mathrm{Am}$ Coll Cardiol. 2006;47:789-793.

31. Manovel A, Dawson D, Smith B, Nihoyannopoulos P. Assessment of left ventricular function by different speckle-tracking software. Eur J Echocardiogr. 2010;11:417-421.

32. Belghitia H, Brette S, Lafitte S, Reant P, Picard F, Serri K, et al. Automated function imaging: a new operator-independent strain method for assessing left ventricular function. Arch Cardiovasc Dis. 2008;101(3):163-169.

33. Marwick TH. Measurement of strain and strain rate by echocardiography: ready for prime time? J Am Coll Cardiol. 2006;47:1313-1327.

34. Leitman M, Lysyansky P, Sidenko S, Shir V, Peleg E, Binenbaum $M$, et al. Two-dimensional strain-a novel software for realtime quantitative echocardiographic assessment of myocardial function. J Am Soc Echocardiogr. 2004;17(10):1021-1029.

35. Mor-Avi V, Lang RM, Badano LP, Belohlavek M, Cardim NM, Derumeaux G, et al. Current and evolving echocardiographic techniques for the quantitative evaluation of cardiac mechanics: ASE/EAE consensus statement on methodology and indications 
endorsed by the Japanese Society of Echocardiography. Eur J Echocardiogr. 2011;12(3):167-205.

36. Voigt JU, Pedrizzetti G, Lysyansky P, Marwick TH, Houle H, Baumann $R$, et al. Definitions for a common standard for 2D speckle tracking echocardiography: consensus document of the EACVI/ASE/Industry Task Force to standardize deformation imaging. Eur Heart J Cardiovasc Imaging. 2015;16(1):1-11.

37. Duncan AE, Alfirevic A, Sessler DI, Popovic ZB, Thomas JD. Perioperative assessment of myocardial deformation. Anesth Analg. 2014;118(3):525-544.

38. Notomi Y, Lysyansky P, Setser RM, Shiota T, Popović ZB, Martin-Miklovic MG, et al. Measurement of ventricular torsion by two-dimensional ultrasound speckle tracking imaging. J Am Coll Cardiol. 2005;45(12):2034-2041.

39. Pavlopoulos H, Nihoyannopoulos P. Strain and strain rate deformation parameters: from tissue Doppler to 2D speckle tracking. Int J Cardiovasc Imaging. 2008;24:479-491.

40. Sengupta PP, Krishnamoorthy VK, Korinek J, Narula J, Vannan MA, Lester SJ, et al. Left ventricular form and function revisited: applied translational science to cardiovascular ultrasound imaging. J Am Soc Echocardiogr. 2007;20(5):539-551.

41. Teske AJ, De Boeck B, Melman PG, Sieswerda GT, Doevendas PA, Cramer JM. Echocardiographic quantification of myocardial function using tissue deformation imaging, a guide to image acquisition and analysis using tissue Doppler and speckle tracking. Cardiovasc Ultrasound. 2007;5:27.

42. Marwick TH, Leano RL, Brown J, Sun JP, Hoffmann R, Lysyansky $P$, et al. Myocardial strain measurement with 2-dimensional speckle-tracking echocardiography: definition of normal range. JACC Cardiovasc Imaging. 2009;2(1):80-84.

43. Yingchoncharoen T, Agarwal S, Popović ZB, Marwick TH. Normal ranges of left ventricular strain: a meta-analysis. $J \mathrm{Am}$ Soc Echocardiogr. 2013;26(2):185-191.

44. Cheng S, Larson MG, McCabe EL, Osypiuk E, Lehman BT, Stanchev $P$, et al. Age- and sex-based reference limits and clinical correlates of myocardial strain and synchrony: the Framingham Heart Study. Circ Cardiovasc Imaging. 2013;6(5):692-699.

45. Kuznetsova T, Herbots L, Richart T, D'hooge J, Thijs L, Fagard $\mathrm{RH}$, et al. Left ventricular strain and strain rate in a general population. Eur Heart J. 2008;29:2014-2023.

46. Dalen H, Thorstensen A, Aase SA, Ingul CB, Torp H, Vatten LJ, et al. Segmental and global longitudinal strain and strain rate based on echocardiography of 1266 healthy individuals: the HUNT study in Norway. Eur J Echocardiogr. 2010;11:176-183.

47. Sun JP, Lee AP, Wu C, Lam YY, Hung MJ, Chen L, et al. Quantification of left ventricular regional myocardial function using two-dimensional speckle tracking echocardiography in healthy volunteers - a multi-center study. Int J Cardiol. 2013;167:495-501.

48. Fonseca CG, Oxenham HC, Cowan BR, Occleshaw CJ, Young AA. Aging alters patterns of regional nonuniformity in LV strain relaxation: a 3-D MR tissue tagging study. Am J Physiol Heart Circ Physiol. 2003;285:621-630.

49. Hurlburt HM, Aurigemma GP, Hill JC, Narayanan A, Gaasch WH, Vinch CS, et al. Direct ultrasound measurement of longitudinal, circumferential, and radial strain using 2-dimensional strain imaging in normal adults. Echocardiography. 2007;24(7):723-731.

50. Rosner A, Bijnens B, Hansen M, How OJ, Aarsaether E, Muller S, et al. Left ventricular size determines tissue Doppler-derived longitudinal strain and strain rate. Eur J Echocardiogr. 2009;10:271-277.

51. Ferferieva V, Van den Bergh A, Claus P, Jasaityte R, Veulemans $\mathrm{P}$, Pellens $\mathrm{M}$, et al. The relative value of strain and strain rate for defining intrinsic myocardial function. Am J Physiol Heart Circ Physiol. 2012;302(1):H188-H195.

52. Weidemann F, Jamal F, Kowalski M, Kukulski T, D'Hooge J, Bijnens $\mathrm{B}$, et al. Can strain rate and strain quantify changes in regional systolic function during dobutamine infusion, B-blockade, and atrial pacing -implications for quantitative stress echocardiography. J Am Soc Echocardiogr. 2002;15:416-424.

53. Kovács A, Oláh A, Lux Á, Mátyás C, Németh BT, Kellermayer D, et al. Strain and strain rate by speckle-tracking echocardiography correlate with pressure-volume loop-derived contractility indices in a rat model of athlete's heart. Am J Physiol Heart Circ Physiol. 2015;308(7):H743-H748.

54. Rodríguez-Bailón I, Jiménez-Navarro MF, Pérez-González R, García-Orta R, Morillo-Velarde E, de Teresa-Galván E. Deformación ventricular izquierda en ecocardiografía bidimensional: valores y tiempos en sujetos normales. Rev Esp Cardiol. 2010;63(10):1195-1199.

55. Landesberg G, Jaffe AS, Gilon D, Levin PD, Goodman S, AbuBaih A, et al. Troponin elevation in severe sepsis and septic shock: the role of left ventricular diastolic dysfunction and right ventricular dilatation. Crit Care Med. 2014;42(4):790-800.

56. Orde SR, Pulido JN, Masaki M, Gillespie S, Spoon JN, Kane GC, et al. Outcome prediction in sepsis: speckle tracking echocardiography based assessment of myocardial function. Crit Care. 2014;18(4):R149.

57. De Geer L, Engvall J, Oscarsson A. Strain echocardiography in septic shock - a comparison with systolic and diastolic function parameters, cardiac biomarkers and outcome. Crit Care. 2015;19:122.

58. Chang WT, Lee WH, Lee WT, Chen PS, Su YR, Liu PY, et al. Left ventricular global longitudinal strain is independently associated with mortality in septic shock patients. Intensive Care Med. 2015;41(10):1791-1799.

59. Palmieri V, Innocenti F, Guzzo A, Guerrini E, Vignaroli D, Pini R. Left ventricular systolic longitudinal function as predictor of outcome in patients with sepsis. Circ Cardiovasc Imaging. 2015;8(11):e003865.

60. Ng PY, Sin WC, Ng AK, Chan WM. Speckle tracking echocardiography in patients with septic shock: a case control study (SPECKSS). Crit Care. 2016;20(1):145.

61. Zaky A, Gill EA, Lin CP, Paul CP, Bendjelid K, Treggiari MM. Characteristics of sepsis-induced cardiac dysfunction using speckle-tracking echocardiography: a feasibility study. Anaesth Intensive Care. 2016;44(1):65-76.

62. De Geer L, Oscarsson A, Engvall J. Variability in echocardiographic measurements of left ventricular function in septic shock patients. Cardiovasc Ultrasound. 2015;13:19.

63. Dalla K, Hallman C, Bech-Hanssen O, Haney M, Ricksten SE. Strain echocardiography identifies impaired longitudinal systolic function in patients with septic shock and preserved ejection fraction. Cardiovasc Ultrasound. 2015;13:30.

64. Kalam K, Otahal P, Marwick TH. Prognostic implications of global LV dysfunction: a systematic review and meta-analysis of global longitudinal strain and ejection fraction. Heart. 2014;100(21):1673-1680.

65. Innocenti F, Palmieri V, Guzzo A, Stefanone VT, Donnini C, Pini $R$. SOFA score and left ventricular systolic function as predictors of short-term outcome in patients with sepsis. Intern Emerg Med. 2018;13(1):51-58.

66. Lang RM, Badano LP, Mor-Avi V, Afilalo J, Armstrong A, Ernande $\mathrm{L}$, et al. Recomendaciones para la cuantificación de las cavidades cardiacas por ecocardiografía en adultos: actualización de la Sociedad Americana de Ecocardiografía y de la Asociación Europea de Imagen Cardiovascular. Asociación de Ecocardiografía de la Sociedad Interamericana de Cardiología (ECOSIAC); 2018.

67. Liu YW, Su CT, Huang YY, Yang CS, Huang JW, Yang MT, et al. Left ventricular systolic strain in chronic kidney disease and hemodialysis patients. Am J Nephrol. 2011;33(1):84-90.

Conflicto de intereses: Los autores reportan que no hay conflicto de intereses. No se requirió financiamiento externo.

Correspondencia:

Ahtziri Yunuén Rodríguez Santos

Calle 27, Número 57, Departamento 402,

Colonia San Pedro de los Pinos,

Del. Benito Juárez, 03800.

E-mail: hatz5@hotmail.com 